\title{
28 Research Square \\ Efficacy of PD-1/PD-L1 Inhibitors in Ovarian Cancer: A Single-arm Meta-analysis
}

\section{Jue Zhu}

Ningbo Women and Children's Hospital

Lifeng Yan

Ningbo Women and Children's Hospital

Qiming Wang ( $D$ nbfewqm@126.com )

Ningbo Women and Children's Hospital

\section{Research}

Keywords: PD-1/PD-L1 inhibitors, immunotherapy, ovarian cancer

Posted Date: May 4th, 2021

DOI: https://doi.org/10.21203/rs.3.rs-412259/v1

License: (1) This work is licensed under a Creative Commons Attribution 4.0 International License. Read Full License 


\section{Abstract}

Several studies have evaluated the efficacy of PD-1/PD-L1 inhibitors in ovarian cancer; however, the response rate varies. This study aims to explore the efficacy of anti-PD-1/PD-L1 therapy in ovarian cancer. A quantitative meta-analysis was performed through a systematic search in PubMed, Web of Science, and the Cochrane Library. The pooled ORR was calculated and compared. Fifteen trials were included in this meta-analysis. Our analyses showed that the pooled ORR of all included studies was $19 \%$ (95\% Cl: 13\%, 27\%). Single PD-1/PD-L1 inhibitors had the lowest ORR of 9\% (95\% Cl: 7\%, 12\%), while the combination of PD-1/PD-L1 inhibitors and chemotherapy had the highest ORR of $36 \%$ (95\% Cl: $24 \%$, $51 \%)$. This study showed that PD-1/PD-L1 inhibitors alone have limited efficacy for ovarian cancer. The combination of PD-1/PD-L1 inhibitors and chemotherapy could be chosen as the recommended modality for further study.

\section{Background}

Ovarian cancer (OC) is the eighth most common cancer in women worldwide (tenth in China) (1). Due to its insidious onset and vague presenting symptoms, almost two-thirds of patients are diagnosed with advanced disease (2), which is associated with significant mortality. The 5-year survival rate ranges from $35-45.6 \%$ in patients with advanced-stage disease (3). Platinum/taxane-based chemotherapy with or without bevacizumab is still the standard of care for advanced OC. Currently, the overall response rate (ORR) of primary treatment is $60-80 \%$; however, $70 \%$ of patients relapse within 5 years, and many of them develop drug-resistant disease (4). Poly ADP-ribose polymerase (PARP) inhibitors are shifting the paradigm of care for $\mathrm{OC}$ patients. Nevertheless, new strategies are still needed for these patients.

Immune checkpoint inhibitor therapies have transformed cancer treatment in various solid malignant tumors, such as melanoma, non-small cell lung cancer, liver cancer, and renal cell carcinomas. In particular, anti-PD-1 or PD-L1 therapy is becoming increasingly popular in cancer therapy. In contrast to traditional chemotherapy or targeted therapy, immunotherapy shows a clear plateau in the overall survival curve, representing long-term survivors. Currently, a series of phase I/II studies have evaluated the efficacy of anti-PD-1 or PD-L1 therapy in OC, with ORR ranging from $8-60 \%$ and median progression-free survival (PFS) times ranging from 2 to 10 months (5-7). The quite different responses to anti-PD-1 or PDL1 therapy might be attributed to different combination therapies or OC types. Therefore, it is necessary to investigate specific combination therapies or subtypes of $\mathrm{OC}$ that benefit most from immunotherapy. Most of these trials were designed as single-arm trials and had noncomparable forms. Therefore, we conducted this quantitative meta-analysis to explore the efficacy of anti-PD-1/PD-L1 therapy in OC.

\section{Methods}

\subsection{Search strategy}


We searched PubMed, Web of Science, and the Cochrane Library from 1966 to January 19, 2021. We also reviewed records of the American Society of Clinical Oncology (ASCO) and the European Society for Medical Oncology (ESMO). The following search terms were used: "PD-L1", "PD-1", "pembrolizumab”, "nivolumab", "atezolizumab", "durvalumab", "avelumab", and "ovarian cancer". The references of literature reviews and original articles were also scanned to avoid missing any qualified studies.

\subsection{Inclusion and exclusion criteria}

The inclusion criteria were as follows: (1) prospective clinical studies (including randomized control trials and single-arm studies); (2) articles investigating PD-1/PD-L1 inhibitors in OC patients; and (3) studies reporting the overall response rate (ORR). The exclusion criteria were as follows: (1) article type: letters, editorials, expert opinions, case reports and reviews; (2) studies without usable data; and (3) duplicate publications.

\subsection{Data extraction}

Two investigators extracted data from the eligible studies independently, and any disagreements were resolved by discussion with a third investigator. For each study, the following characteristic information was recorded: first author, year of publication, number of patients, ORR, disease control rate (DCR), therapeutic regimen, and response to previous platinum-based regimen.

\subsection{Quality assessment}

Most of the included studies were single arm or non-controlled studies. Therefore, the Newcastle-Ottawa Scale (NOS) tools were used to assess the quality of included studies (8). Studies with more than 4 stars were included for further analysis. Any discrepancies were resolved by consensus.

\subsection{Statistical analysis}

Statistical analyses of the pooled ORR were performed using $\mathrm{R}$ version 3.5.2. The heterogeneity of the data was evaluated by chi-square $Q$ test and $I^{2}$ statistic. For the $Q$ test, a $p$ value less than 0.05 indicated significant heterogeneity; for the $\mathrm{I}^{2}$ statistics, an $\mathrm{I}^{2}$ value greater than $50 \%$ was considered significant heterogeneity. Meta-regression and subgroup analyses were performed to identify the factor contributing risk of bias.

\section{Results}

\subsection{Patient characteristics}

The initial search yielded 430 records. After screening the titles and abstracts, 35 full-text articles were assessed for eligibility. Finally, a total of 15 articles were included in this study $(5-7,9-20)$. The study selection process is shown in Fig. 1. Among these 15 studies, the administered PD-1/PD-L1 inhibitors were pembrolizumab (6), nivolumab (3), durvalumab (3), atezolizumab (2), and avelumab (1). Thirteen of 
15 studies were assessed as $7-9$ stars, and 2 were assessed as 5 stars. The details are summarized in Table 1.

Table 1

Characteristics of 15 included studies

\begin{tabular}{|c|c|c|c|c|c|}
\hline First Author & Year & Total & Interventions & $\begin{array}{l}\text { Platinum- } \\
\text { resistant }\end{array}$ & $\begin{array}{l}\text { NOS } \\
\text { score }\end{array}$ \\
\hline Hamanishi (9) & 2015 & 20 & Nivolumab & Yes & 8 \\
\hline Disis (10) & 2019 & 125 & Avelumab & NA & 8 \\
\hline $\begin{array}{l}\text { Konstantinopoulos } \\
\text { (11) }\end{array}$ & 2019 & 60 & Pembrolizumab + niraparib & Yes & 8 \\
\hline Liu (12) & 2019 & 9 & Atezolizumab & NA & 8 \\
\hline Liu (13) & 2019 & 38 & Nivolumab + bevacizumab & NA & 8 \\
\hline Matulonis (5) & 2019 & 376 & Pembrolizumab & NA & 8 \\
\hline Varga (14) & 2019 & 26 & Pembrolizumab & NA & 8 \\
\hline Walsh (6) & 2019 & 14 & $\begin{array}{l}\text { Pembrolizumab + cisplatin + } \\
\text { gemcitabine }\end{array}$ & Yes & 5 \\
\hline Lampert (15) & 2020 & 35 & Durvaluamb + olaparib & NA & 7 \\
\hline Lee (16) & 2020 & 23 & $\begin{array}{l}\text { Pembrolizumab + pegylated } \\
\text { liposomal doxorubicin }\end{array}$ & Yes & 7 \\
\hline Moroney (17) & 2020 & 12 & Atezolizumab + bevacizumab & Yes & 7 \\
\hline O'Cearbhaill (18) & 2020 & 40 & $\begin{array}{l}\text { Durvalumab + pegylated liposomal } \\
\text { doxorubicin }\end{array}$ & Yes & 5 \\
\hline Zamarin (19) & 2020 & 27 & $\begin{array}{l}\text { Pembrolizumab + folate receptor } \\
\text { alpha vaccine }\end{array}$ & Yes & 9 \\
\hline Zamarin (20) & 2020 & 100 & $\begin{array}{l}\text { Nivolumab + ipilimumab vs } \\
\text { nivolumab }\end{array}$ & NA & 7 \\
\hline Zsiros (7) & 2020 & 40 & $\begin{array}{l}\text { Pembrolizumab + bevacizumab + } \\
\text { cyclophosphamide }\end{array}$ & NA & 8 \\
\hline
\end{tabular}

\subsection{Efficacy}

All included studies reported the ORR as the clinical activity outcome. The ORRs across the studies varied from $4-48 \%$. The random-effects model was adopted because of significant heterogeneity $\left(I^{2}=81 \%, p<\right.$ 0.01). The analysis showed a pooled ORR of $19 \%$ (95\% Cl: 13\%, 27\%) (Fig. 2). As significant heterogeneity 
in the ORR existed across the studies, meta-regression and subgroup analyses were performed to explore the potential sources of heterogeneity.

\subsection{Meta-regression and subgroup analyses}

Previous studies showed single PD-1/PD-L1 inhibitors had limited response rate. Additional, platinumresistant $\mathrm{OC}$ had poor response to subsequent therapy. Therefore, regimen combination and platinumresistant status were included for meta-regression analysis. The results showed immunotherapy regimen combination (single PD-1/PD-L1 inhibitor vs. combination of PD-1/PD-L1 inhibitor with other anti-tumor drugs, $p<0.003$ ) contributed to heterogeneity of ORR, while whether platinum-sensitive or -resistance did not influence ORR.

\subsubsection{ORR in Different PD-1/PD-L1 inhibitor combinations}

Six studies on a single PD-1/PD-L1 inhibitor had usable ORR data. The pooled ORR was 9\% (95\% Cl: 7\%, $12 \%)$ without significant heterogeneity $\left(I^{2}=0 \%, p=0.58\right)$ (Fig. 3A). Four studies on a combination of PD1/PD-L1 inhibitors and chemotherapy reported ORR data. The pooled ORR was 36\% (95\% Cl: 24\%, 51\%), and significant heterogeneity existed $\left(\mathrm{I}^{2}=66 \%, \mathrm{p}=0.03\right)$ (Fig. 3B). Two studies on a combination of PD1/PD-L1 inhibitors and antiangiogenic therapy reported ORR data. The pooled ORR was 30\% (95\% Cl: $19 \%, 44 \%)$ without significant heterogeneity $\left(I^{2}=0 \%, p=0.67\right)$ (Fig. 3C). Two studies on a combination of PD-1/PD-L1 inhibitors and PARP inhibitors reported ORR data. The pooled ORR was 17\% (95\% Cl: 11\%, $26 \%)$ without significant heterogeneity $\left(I^{2}=0 \%, p=0.61\right)$ (Fig. 3D).

\subsubsection{ORR of platinum-resistant and platinum-sensitive patients}

Eleven studies with a total of 594 patients reported the ORR according to prior treatment response (platinum-resistant and platinum-sensitive). The pooled ORR was 21\% (95\% Cl: 14\%, 31\%) with significant heterogeneity $\left(\mathrm{I}^{2}=79 \%, \mathrm{p}<0.01\right)$ (Fig. 4). In 11 studies on platinum-resistant patients, the pooled ORR was $19 \%$ (95\% Cl: 12\%, 28\%) with significant heterogeneity $\left(I^{2}=73 \%, p<0.01\right)$. In 4 studies on platinumsensitive patients, the pooled ORR was 31\% (95\% Cl: $12 \%, 61 \%)$ with significant heterogeneity $\left(\mathrm{I}^{2}=89 \%, \mathrm{p}\right.$ $<0.01$ ) (Fig. 4).

In light of the poor treatment response of single PD-1/PD-L1 inhibitors, we performed meta-analyses separately in platinum-resistant and platinum-sensitive patients, excluding those treatment arms with a single PD-1/PD-L1 inhibitor. In 8 studies on platinum-resistant patients, the pooled ORR was 25\% (95\% Cl: $17 \%, 35 \%)$ with significant heterogeneity $\left(I^{2}=59 \%, p=0.02\right.$; Fig. $\left.5 A\right)$. In 3 studies on platinum-sensitive patients, the pooled ORR was $49 \%(95 \% \mathrm{Cl}: 33 \%, 65 \%)$ without significant heterogeneity $\left(\mathrm{I}^{2}=0 \%, \mathrm{p}<0.68\right.$; Fig. 5B).

\subsection{Publication bias}


The funnel plot for the ORR of the included studies was roughly symmetric (Fig. 6). We also performed Egger's and Begg's tests to assess the presence of publication bias in this study. No significantly different results emerged, with $p=0.331$ for Egger's test and $p=0.656$ for Begg's test.

\section{Discussion}

This study included 15 clinical trials involving 945 patients to evaluate the efficacy of PD-1/PD-L1 inhibitors in treating advanced OC. The pooled results showed that the ORR was 19\%. Single PD-1/PD-L1 inhibitors showed limited efficacy, with an ORR of $9 \%$, while combination with chemotherapy showed an increased ORR of $36 \%$. In addition, PD-1/PD-L1 inhibitors had a higher ORR in platinum-sensitive OC than in platinum-resistant OC (31\% vs $19 \%)$.

Immune checkpoint inhibitors, especially PD-1/PD-L1 inhibitors, are changing the treatment paradigm in certain cancers, such as melanoma and non-small cell lung cancer. The overall ORR with single PD-1/PDL1 inhibitors across other cancers was approximately $20 \%$, while it was $9 \%$ in OC. Previous studies have shown that PD-L1 expression (tumor cells and/or tumor-infiltrating lymphocytes), tumor mutational burden (TMB), microsatellite instability (MSI) and/or mismatch repair (MMR) deficiency are effective predictive biomarkers for anti-PD1/PD-L1 therapy. However, KEYNOTE-028 showed a poor ORR for PD-1 inhibitors, even in PD-L1-positive OC patients (14). Additionally, KEYNOTE-100 showed a low rate of MSI in OC. As for TMB, it was also very low in OC patients. Therefore, seeking an optimal treatment modality with PD-1/PD-L1 inhibitors seems necessary before identifying a better predictive biomarker.

Vascular endothelial growth factor (VEGF) creates an immunosuppressive microenvironment within cancers by suppressing dendritic cell maturation, increasing the Treg population and stimulating the growth of myeloid-derived suppressor cells in the tumor microenvironment $(21,22)$. Bevacizumab can reverse these VEGF-mediated immunosuppressive effects on the tumor microenvironment, potentially augmenting immune-mediated antitumor activity. Several studies have demonstrated the synergistic effect between antiangiogenic agents and PD-1/PD-L1 inhibitors in solid tumors, including renal cancer, non-small lung cancer, and endometrial cancer (23-27). OC is known to highly express VEGF, which serves as a major driver of tumor neovascularization and local immune suppression (28). Therefore, antiVEGF agents could theoretically enhance the efficacy of immunotherapy in OC. This study also showed a high ORR of $30 \%$ in OC patients treated with antiangiogenic agents and PD-1/PD-L1 inhibitors.

In recent years, increasing evidence has shown that chemotherapy is not only a cytotoxic agent but also a stimulator of tumor-specific immune responses. Chemotherapy involves the stimulation of anticancer immunity either by initiating the release of immunostimulatory molecules from dying cancer cells or by mediating off-target effects on immune cell populations (29). On the one hand, chemotherapy could induce immunogenic cell death (ICD), enabling the release of neoantigens and signals to antigenpresenting cells; on the other hand, chemotherapy was found to reduce the number and activity of immune-suppressive cells, including myeloid-derived suppressor cells and Treg cells (30-32). Therefore, chemotherapy can theoretically initiate or restore anticancer immune responses by converting 
immunologically "cold" tumors into "hot" tumors. Several studies have shown clinical activities with a combination of immunotherapy and chemotherapy $(33,34)$. This study also showed a high ORR of $36 \%$ in OC patients treated with chemotherapy and PD-1/PD-L1 inhibitors.

Platinum-resistant $\mathrm{OC}$ is a dismal disease and has a low response to subsequent chemotherapy. In this study, we found that the pooled ORR was $25 \%$ in studies on platinum-resistant patients and $49 \%$ in studies on platinum-sensitive patients. This might be attributed to the immunosuppressive environment in platinum-resistant OC. Data on the tumor microenvironment of platinum-resistant OC showed low CD8 $+T$ cell infiltration and highly activated CD4 + T cells $(9,35)$.

This study had some limitations. First, most of the included articles were noncomparable studies, and some of them had small sample sizes. Second, the PD-1/PD-L1 inhibitors were different among studies, which inevitably caused bias. Third, the complete data were hardly accessible in some studies to perform subgroup analysis.

\section{Conclusions}

We believe that conducting this meta-analysis was timely and necessary. PD-1/PD-L1 inhibitors alone have limited efficacy for OC. Combination with other therapeutics might be a promising treatment option. The combination of PD-1/PD-L1 inhibitors and chemotherapy showed the highest ORR and could be chosen as the recommended modality for further study.

\section{List Of Abbreviations}

\begin{tabular}{|ll|}
\hline PM & peritoneal carcinomatosis \\
OC & ovarian cancer \\
ORR & overall response rate \\
PARP & Poly ADP-ribose polymerase \\
PFS & progression-free survival \\
\hline
\end{tabular}

\section{Declarations}

- Ethics approval and consent to participate

None.

- Consent for publication

All authors agreed publication of the manuscript. 
The datasets used and/or analyzed during the current study are available from the corresponding author on reasonable request.

\section{- Competing interests}

The authors have stated that they have no conflicts of interest.

\section{- Funding}

This study was supported by grants from Scientific research funds of Zhejiang province health department (2021KY1055). The funders had no role in study design, data collection and analysis, decision to publish, or preparation of the manuscript.

\section{- Authors' contributions}

Writing original draft and editing: JZ. Methodology and data acquisition: LY; data analyses and interpretation: JZ and QW; study concept and design, project administration: QW; Manuscript revision: JZ and QW. All authors read and approved the final manuscript. All authors read and approved the final manuscript.

\section{- Acknowledgements}

None.

\section{References}

1. Sung H, Ferlay J, Siegel RL, Laversanne M, Soerjomataram I, Jemal A, et al. Global cancer statistics 2020: GLOBOCAN estimates of incidence and mortality worldwide for 36 cancers in 185 countries. CA Cancer J Clin 2021.

2. Jessmon P, Boulanger T, Zhou W, Patwardhan P. Epidemiology and treatment patterns of epithelial ovarian cancer. Expert Rev Anticancer Ther 2017;17:427-37.

3. Oronsky B, Ray CM, Spira Al, Trepel JB, Carter CA, Cottrill HM. A brief review of the management of platinum-resistant-platinum-refractory ovarian cancer. Med Oncol 2017;34:103.

4. Jayson GC, Kohn EC, Kitchener HC, Ledermann JA. Ovarian cancer. Lancet 2014;384:1376-88.

5. Matulonis UA, Shapira-Frommer R, Santin AD, Lisyanskaya AS, Pignata S, Vergote I, et al. Antitumor activity and safety of pembrolizumab in patients with advanced recurrent ovarian cancer: results from the phase II KEYNOTE-100 study. Ann Oncol 2019;30:1080-7.

6. Walsh C, Kamrava M, Rogatko A, Li AJ, Cass I, Rimel B. Phase II trial of pembrolizumab with cisplatin and gemcitabine in women with recurrent platinum-resistant ovarian cancer. Gynecologic Oncology 2019;154:16-7.

7. Zsiros E, Lynam S, Attwood KM, Wang C, Chilakapati S, Gomez EC, et al. Efficacy and Safety of Pembrolizumab in Combination With Bevacizumab and Oral Metronomic Cyclophosphamide in the 
Treatment of Recurrent Ovarian Cancer: A Phase 2 Nonrandomized Clinical Trial. JAMA Oncol 2020.

8. Lo CK, Mertz D, Loeb M. Newcastle-Ottawa Scale: comparing reviewers' to authors' assessments. BMC Med Res Methodol 2014;14:45.

9. Hamanishi J, Mandai M, Ikeda T, Minami M, Kawaguchi A, Murayama T, et al. Safety and Antitumor Activity of Anti-PD-1 Antibody, Nivolumab, in Patients With Platinum-Resistant Ovarian Cancer. J Clin Oncol 2015;33:4015-22.

10. Disis ML, Taylor MH, Kelly K, Beck JT, Gordon M, Moore KM, et al. Efficacy and Safety of Avelumab for Patients With Recurrent or Refractory Ovarian Cancer: Phase 1b Results From the JAVELIN Solid Tumor Trial. JAMA Oncol 2019;5:393-401.

11. Konstantinopoulos PA, Waggoner S, Vidal GA, Mita M, Moroney JW, Holloway R, et al. Single-Arm Phases 1 and 2 Trial of Niraparib in Combination With Pembrolizumab in Patients With Recurrent Platinum-Resistant Ovarian Carcinoma. JAMA Oncol 2019;5:1141-9.

12. Liu JF, Gordon M, Veneris J, Braiteh F, Balmanoukian A, Eder JP, et al. Safety, clinical activity and biomarker assessments of atezolizumab from a Phase I study in advanced/recurrent ovarian and uterine cancers. Gynecol Oncol 2019;154:314-22.

13. Liu JF, Herold C, Gray KP, Penson RT, Horowitz N, Konstantinopoulos PA, et al. Assessment of Combined Nivolumab and Bevacizumab in Relapsed Ovarian Cancer: A Phase 2 Clinical Trial. JAMA Oncol 2019;5:1731-8.

14. Varga A, Piha-Paul S, Ott PA, Mehnert JM, Berton-Rigaud D, Morosky A, et al. Pembrolizumab in patients with programmed death ligand 1-positive advanced ovarian cancer: Analysis of KEYNOTE028. Gynecol Oncol 2019;152:243-50.

15. Lampert EJ, Zimmer A, Padget M, Cimino-Mathews A, Nair JR, Liu Y, et al. Combination of PARP Inhibitor Olaparib, and PD-L1 Inhibitor Durvalumab, in Recurrent Ovarian Cancer: a Proof-of-Concept Phase II Study. Clin Cancer Res 2020;26:4268-79.

16. Lee EK, Xiong N, Cheng SC, Barry WT, Penson RT, Konstantinopoulos PA, et al. Combined pembrolizumab and pegylated liposomal doxorubicin in platinum resistant ovarian cancer: A phase 2 clinical trial. Gynecol Oncol 2020;159:72-8.

17. Moroney JW, Powderly J, Lieu CH, Bendell JC, Eckhardt SG, Chang CW, et al. Safety and Clinical Activity of Atezolizumab Plus Bevacizumab in Patients with Ovarian Cancer: A Phase Ib Study. Clin Cancer Res 2020;26:5631-7.

18. O'Cearbhaill RE, Homicsko K, Wolfer A, DiSilvestro PA, O'Malley DM, Sabbatini P, et al. A phase I/II study of chemo-immunotherapy with durvalumab (durva) and pegylated liposomal doxorubicin (PLD) in platinum-resistant recurrent ovarian cancer (PROC): Genomic sequencing and updated efficacy results. Gynecologic Oncology 2020;159:41.

19. Zamarin D, Burger RA, Sill MW, Powell DJ, Jr., Lankes HA, Feldman MD, et al. Randomized Phase II Trial of Nivolumab Versus Nivolumab and Ipilimumab for Recurrent or Persistent Ovarian Cancer: An NRG Oncology Study. J Clin Oncol 2020;38:1814-23. 
20. Zamarin D, Walderich S, Holland A, Zhou Q, lasonos AE, Torrisi JM, et al. Safety, immunogenicity, and clinical efficacy of durvalumab in combination with folate receptor alpha vaccine TPIV200 in patients with advanced ovarian cancer: a phase II trial. J Immunother Cancer 2020;8.

21. Voron T, Marcheteau E, Pernot S, Colussi O, Tartour E, Taieb J, et al. Control of the immune response by pro-angiogenic factors. Front Oncol 2014;4:70.

22. Yi M, Jiao D, Qin S, Chu Q, Wu K, Li A. Synergistic effect of immune checkpoint blockade and antiangiogenesis in cancer treatment. Mol Cancer 2019;18:60.

23. Motzer RJ, Penkov K, Haanen J, Rini B, Albiges L, Campbell MT, et al. Avelumab plus Axitinib versus Sunitinib for Advanced Renal-Cell Carcinoma. N Engl J Med 2019;380:1103-15.

24. Rini Bl, Plimack ER, Stus V, Gafanov R, Hawkins R, Nosov D, et al. Pembrolizumab plus Axitinib versus Sunitinib for Advanced Renal-Cell Carcinoma. N Engl J Med 2019;380:1116-27.

25. McDermott DF, Huseni MA, Atkins MB, Motzer RJ, Rini BI, Escudier B, et al. Clinical activity and molecular correlates of response to atezolizumab alone or in combination with bevacizumab versus sunitinib in renal cell carcinoma. Nat Med 2018;24:749-57.

26. Reck M, Mok TSK, Nishio M, Jotte RM, Cappuzzo F, Orlandi F, et al. Atezolizumab plus bevacizumab and chemotherapy in non-small-cell lung cancer (IMpower150): key subgroup analyses of patients with EGFR mutations or baseline liver metastases in a randomised, open-label phase 3 trial. Lancet Respir Med 2019;7:387-401.

27. Makker V, Rasco D, Vogelzang NJ, Brose MS, Cohn AL, Mier J, et al. Lenvatinib plus pembrolizumab in patients with advanced endometrial cancer: an interim analysis of a multicentre, open-label, singlearm, phase 2 trial. Lancet Oncol 2019;20:711-8.

28. Fukumura D, Kloepper J, Amoozgar Z, Duda DG, Jain RK. Enhancing cancer immunotherapy using antiangiogenics: opportunities and challenges. Nat Rev Clin Oncol 2018;15:325-40.

29. Galluzzi L, Humeau J, Buqué A, Zitvogel L, Kroemer G. Immunostimulation with chemotherapy in the era of immune checkpoint inhibitors. Nat Rev Clin Oncol 2020;17:725-41.

30. Wang Z, Till B, Gao Q. Chemotherapeutic agent-mediated elimination of myeloid-derived suppressor cells. Oncoimmunology 2017;6:e1331807.

31. Roselli M, Cereda V, di Bari MG, Formica V, Spila A, Jochems C, et al. Effects of conventional therapeutic interventions on the number and function of regulatory $T$ cells. Oncoimmunology 2013;2:e27025.

32. Sevko A, Michels T, Vrohlings M, Umansky L, Beckhove P, Kato M, et al. Antitumor effect of paclitaxel is mediated by inhibition of myeloid-derived suppressor cells and chronic inflammation in the spontaneous melanoma model. J Immunol 2013;190:2464-71.

33. Paz-Ares L, Ciuleanu TE, Cobo M, Schenker M, Zurawski B, Menezes J, et al. First-line nivolumab plus ipilimumab combined with two cycles of chemotherapy in patients with non-small-cell lung cancer (CheckMate 9LA): an international, randomised, open-label, phase 3 trial. Lancet Oncol 2021;22:198211. 
34. Schmid P, Adams S, Rugo HS, Schneeweiss A, Barrios $\mathrm{CH}$, Iwata $\mathrm{H}$, et al. Atezolizumab and NabPaclitaxel in Advanced Triple-Negative Breast Cancer. N Engl J Med 2018;379:2108-21.

35. Mariya T, Hirohashi Y, Torigoe T, Asano T, Kuroda T, Yasuda K, et al. Prognostic impact of human leukocyte antigen class I expression and association of platinum resistance with immunologic profiles in epithelial ovarian cancer. Cancer Immunol Res 2014;2:1220-9.

\section{Figures}




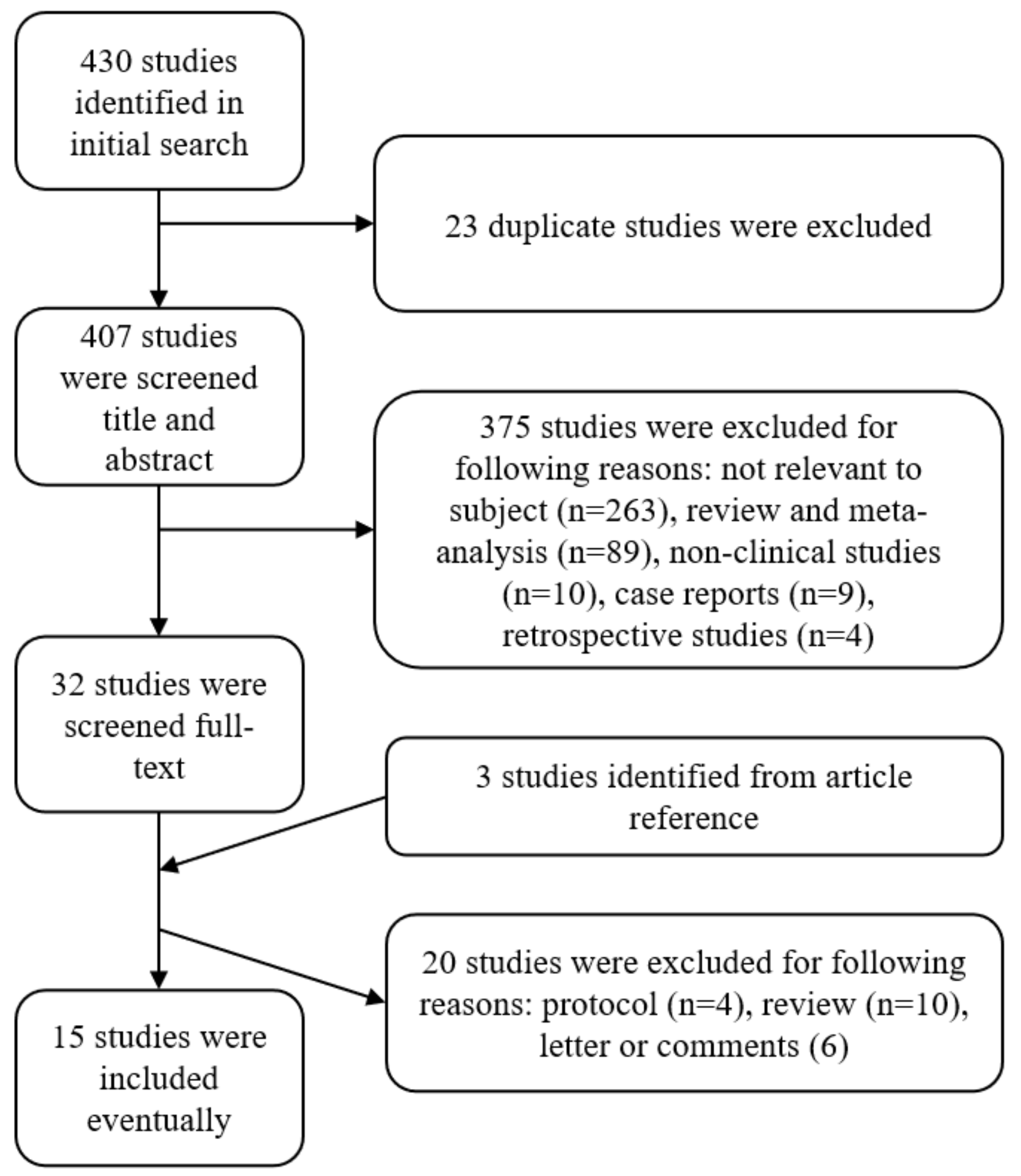

Figure 1

The flow diagram of this meta-analysis 
Hamanishi [9]

Disis [10]

Konstantinopoulos [11]

Liu [12]

Liu [13]

Matulonis [5]

Varga [14]

Walsh [6]

Lampert [15]

Lee [16]

Moroney [17]

O'Cearbhaill [18]

Zamarin [19]

Zamarin [20]

Zsiros [7]

Fixed effect model

Random effects model

Heterogeneity: $I^{2}=81 \%, \tau^{2}=0.4948, p<0.01$

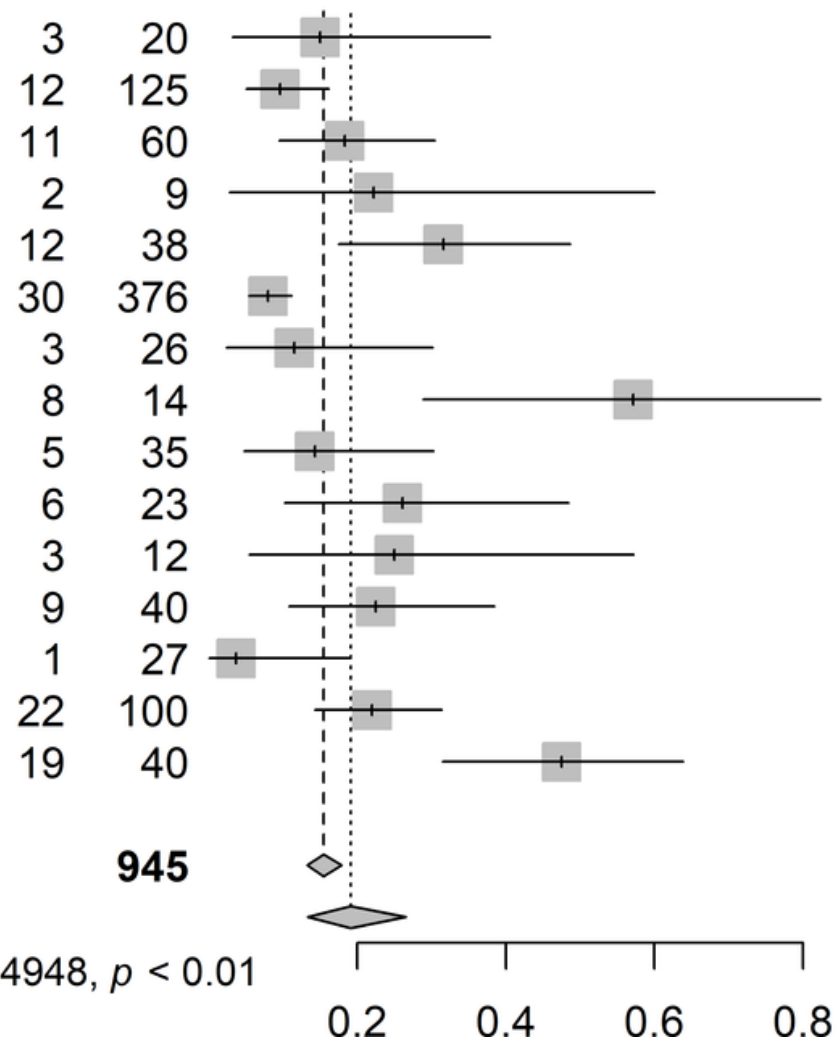

$0.15[0.03 ; 0.38]$

$0.10[0.05 ; 0.16]$

$0.18[0.10 ; 0.30]$

0.22 [0.03; 0.60]

$0.32[0.18 ; 0.49]$

0.08 [0.05; 0.11]

$0.12[0.02 ; 0.30]$

$0.57[0.29 ; 0.82]$

$0.14[0.05 ; 0.30]$

0.26 [0.10;0.48]

$0.25[0.05 ; 0.57]$

$0.22[0.11 ; 0.38]$

$0.04[0.00 ; 0.19]$

$0.22[0.14 ; 0.31]$

0.48 [0.32;0.64]

$0.15[0.13 ; 0.18]$

$0.19[0.13 ; 0.27]$

Figure 2

Summary overall response rate for all included studies. 
Study

Disis [10]

Hamanishi [9]

Liu [12]

Matulonis [5]

Varga [14]

Zamarin [20]

Fixed effect model

Random effects model

Heterogeneity: $I^{2}=0 \%, \tau^{2}=0, p=0.58$
Events Total

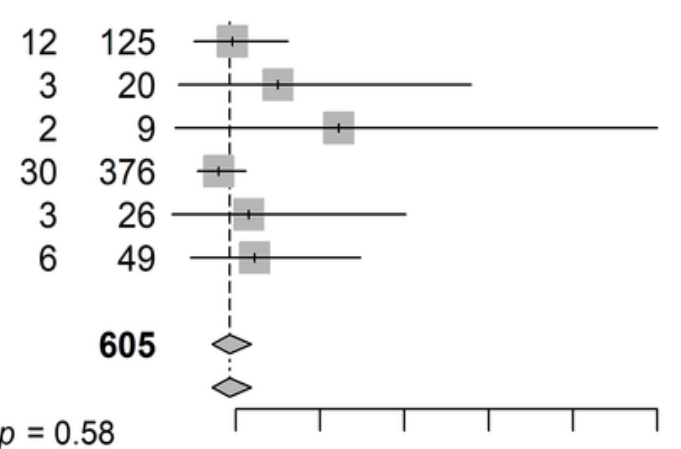

$\begin{array}{llllll}0.1 & 0.2 & 0.3 & 0.4 & 0.5 & 0.6\end{array}$

Proportion $\quad 95 \%-\mathrm{Cl}$

$0.10[0.05 ; 0.16]$

$0.15[0.03 ; 0.38]$

$0.22[0.03 ; 0.60]$

$0.08[0.05 ; 0.11]$

$0.12[0.02 ; 0.30]$

$0.12[0.05 ; 0.25]$

$0.09[0.07 ; 0.12]$

$0.09[0.07 ; 0.12]$

B

Study

Events Total

Proportion $\quad 95 \%-\mathrm{Cl}$

Lee [16]

O'Cearbhaill [18]

Walsh [6]

Zsiros [7]

$\begin{array}{rr}6 & 23 \\ 9 & 40 \\ 8 & 14 \\ 19 & 40\end{array}$

$0.26[0.10 ; 0.48]$

$0.22[0.11 ; 0.38]$

$0.57[0.29 ; 0.82]$

$0.48[0.32 ; 0.64]$

Fixed effect model

117

Random effects model

Heterogeneity: $I^{2}=66 \%, \tau^{2}=0.2091, p=0.03$

$0.36[0.28 ; 0.45]$

$0.36[0.24 ; 0.51]$

C
Study
Events Total
Liu [13]
Moroney [17]

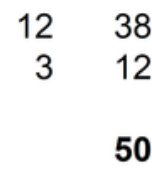
Fixed effect model
Random effects model
Heterogeneity: $I^{2}=0 \%, \tau^{2}=0, p=0.67$

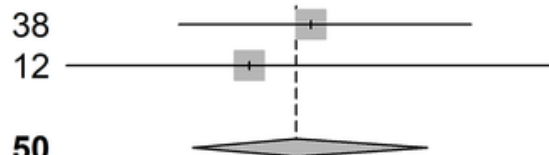

\section{$\begin{array}{lllll}0.1 & 0.2 & 0.3 & 0.4 & 0.5\end{array}$}

Proportion $\quad 95 \%-\mathrm{Cl}$

$0.32[0.18 ; 0.49]$

$0.25[0.05 ; 0.57]$

$0.30[0.19 ; 0.44]$

$0.30[0.19 ; 0.44]$

D

Study

Events Total

Proportion $\quad 95 \%-\mathrm{Cl}$

Konstantinopoulos [11]

Lampert [15]

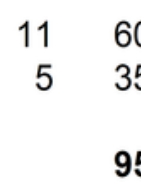

Fixed effect model

95

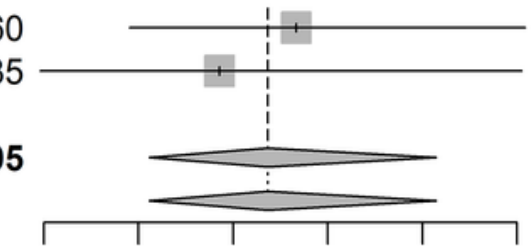

$0.18[0.10 ; 0.30]$

$0.14[0.05 ; 0.30]$

$0.17[0.11 ; 0.26]$

Random effects model

Heterogeneity: $I^{2}=0 \%, \tau^{2}=0, p=0.61$

$\begin{array}{llllll}0.05 & 0.1 & 0.15 & 0.2 & 0.25 & 0.3\end{array}$

Figure 3

Summary overall response rate for different treatment combination. Summary overall response rate for single PD-1/PD-L1 inhibitors (A), PD-1/PD-L1 inhibitors combined with chemotherapy (B), PD-1/PD-L1 inhibitors combined with antiangiogenic therapy (C), and PD-1/PD-L1 inhibitors combined with PARP inhibitors (D). 
Platinum-Resistant $=$ Yes

Hamanishi [9]

Konstantinopoulos [11]

Lampert [15]

Lee [16]

Liu [13]

Matulonis [5]

Moroney [17]

O'Cearbhaill [18]

Walsh [6]

Zamarin [19]

Zsiros [7]

Fixed effect model

Random effects model

Heterogeneity: $I^{2}=73 \%, \tau^{2}=0.5637, p<0.01$

Platinum-Resistant $=$ No

Lampert [15]

Liu [13]

Matulonis [5]

Zsiros [7]

Fixed effect model

Random effects model

Heterogeneity: $I^{2}=89 \%, \tau^{2}=1.1985, p<0.01$

Fixed effect model

594

Random effects model

Heterogeneity: $I^{2}=79 \%, \tau^{2}=0.7802, p<0.01$

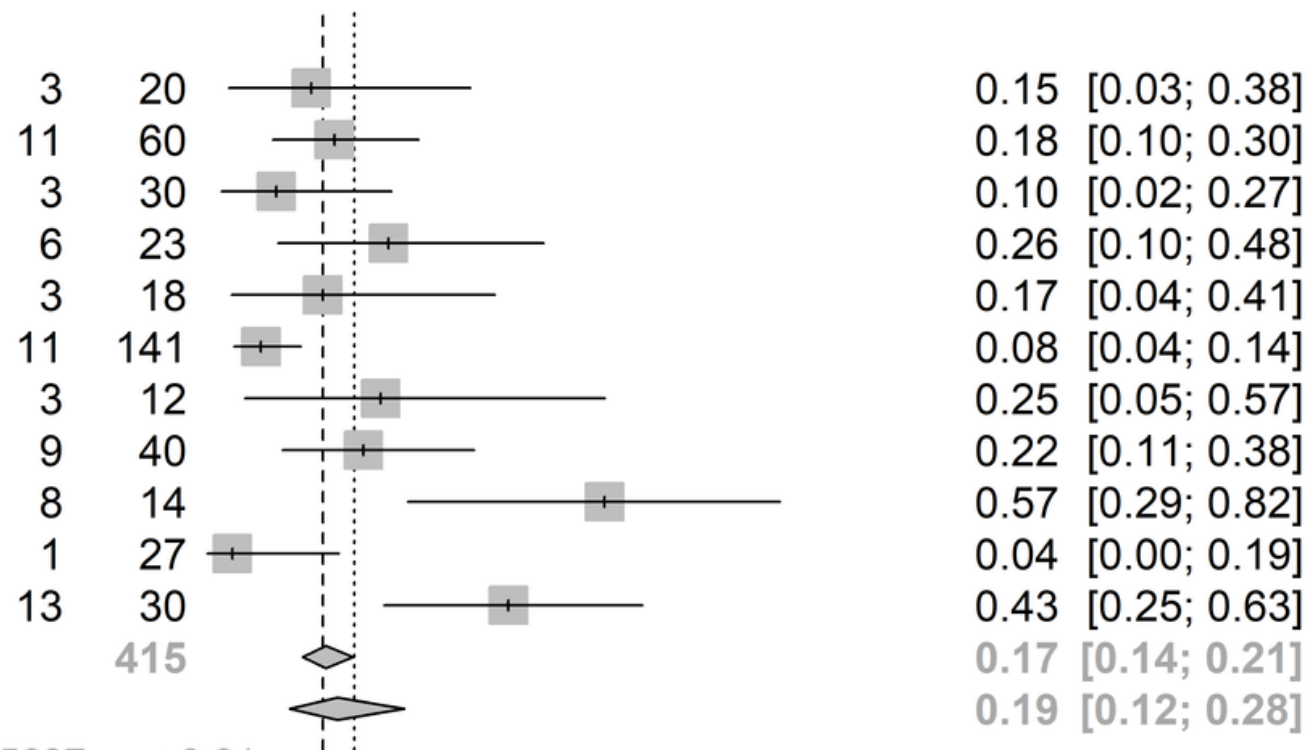

$0.40[0.05 ; 0.85]$

$0.45[0.23 ; 0.68]$

$0.08[0.04 ; 0.13]$

$0.60[0.26 ; 0.88]$

$0.16[0.11 ; 0.22]$

$0.31[0.12 ; 0.61]$

$0.17[0.14 ; 0.20]$

$0.21[0.14 ; 0.31]$

Figure 4

Summary overall response rate for platinum-resistant and platinum-sensitive ovarian cancer. 
A

Study

Konstantinopoulos [11]

Liu [13]

Walsh [6]

Lampert [15]

Lee [16]

Moroney [17]

O'Cearbhaill [18]

Zsiros [7]

Fixed effect model

Random effects model

Heterogeneity: $I^{2}=59 \%, \tau^{2}=0.2742, p=0.02$

Events Total

$\begin{array}{rr}11 & 60 \\ 3 & 18 \\ 8 & 14 \\ 3 & 30 \\ 6 & 23 \\ 3 & 12 \\ 9 & 40 \\ 13 & 30\end{array}$

227

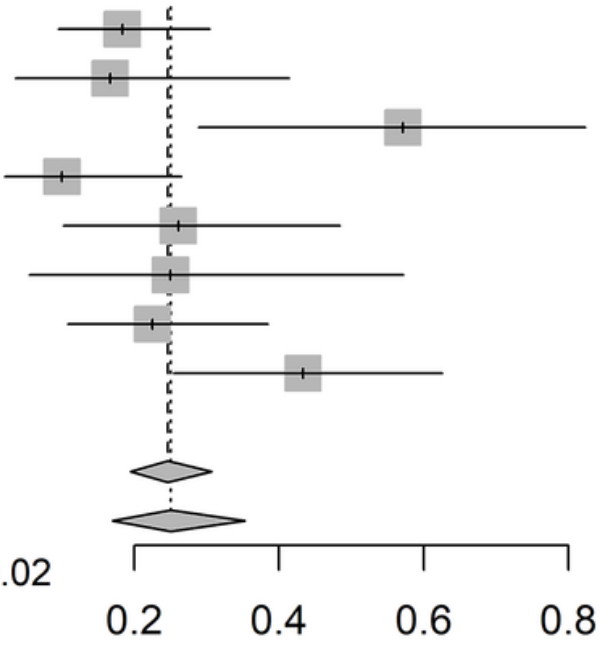

Proportion $\quad 95 \%-\mathrm{Cl}$

$0.18[0.10 ; 0.30]$

$0.17[0.04 ; 0.41]$

$0.57[0.29 ; 0.82]$

$0.10[0.02 ; 0.27]$

$0.26[0.10 ; 0.48]$

$0.25[0.05 ; 0.57]$

$0.22[0.11 ; 0.38]$

$0.43[0.25 ; 0.63]$

$0.25[0.19 ; 0.31]$

$0.25[0.17 ; 0.35]$

B

Study

Liu [13]

Lampert [15]

Zsiros [7]

Fixed effect model

Random effects model

Heterogeneity: $I^{2}=0 \%, \tau^{2}=0, p=0.68$
Events Total

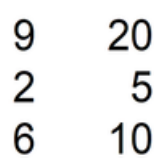

35

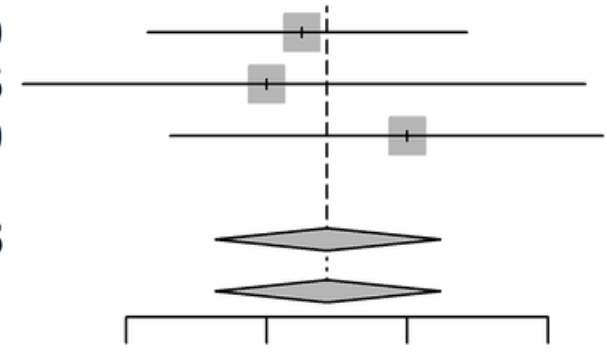

$\begin{array}{llll}0.2 & 0.4 & 0.6 & 0.8\end{array}$
Proportion $\quad 95 \%-\mathrm{Cl}$

$0.45[0.23 ; 0.68]$

$0.40[0.05 ; 0.85]$

$0.60[0.26 ; 0.88]$

$0.49[0.33 ; 0.65]$

$0.49[0.33 ; 0.65]$

Figure 5

Summary overall response rate for platinum-resistant and platinum-sensitive ovarian cancer excluding treatment arms with a single PD-1/PD-L1 inhibitor. 


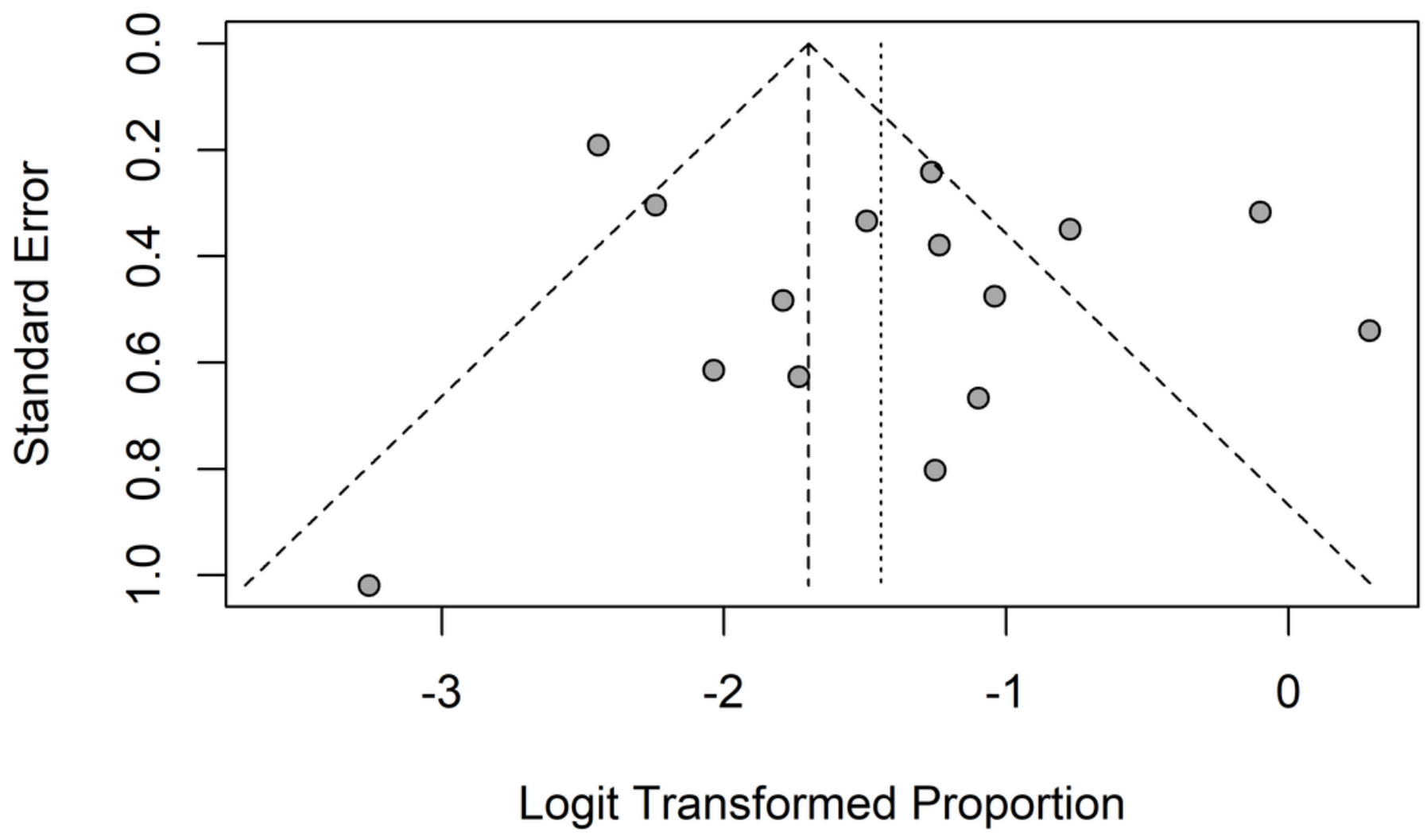

Figure 6

Begg's funnel plot for overall response rate 